\title{
SOME PRODUCTS OF TOPOLOGICAL SPACES WHICH ARE MANIFOLDS
}

\author{
CHARLES D. BASS
}

\begin{abstract}
We give some conditions which imply that a product $X \times Y$ of two metric spaces has the disjoint disks property. As a consequence the products of certain cell-like images of manifolds are shown to be manifolds.
\end{abstract}

1. Introduction. Recent work of Cannon and Edwards, [4], [7], together with unpublished results of F. Quinn have led to simply stated topological criteria for identifying $n$-manifolds. One of these, of particular interest in this paper, is the celebrated "disjoint disks property". A space $X$ is said to have this property if any two maps $f_{1}$ and $f_{2}$ from a 2-cell $D$ into $X$ can be approximated by maps $f_{1}^{\prime}$ and $f_{2}^{\prime}$ respectively from $D$ into $X$ such that $f_{1}^{\prime}(D) \cap f_{2}^{\prime}(D)=\varnothing$. Other authors [1], [3], [8] using the techniques of decomposition space theory have shown that some manifolds may be realized as the product of two spaces $X$ and $Y$ which fail to be manifolds. In this note we will show that certain product spaces have the disjoint disks property, thereby identifying some unusual factorizations of manifolds into nonmanifold factors. This result is Theorem 2 stated in $\$ 3$.

2. Notation. Let $X$ be a metric space, $\rho$ a metric on $X$. For a point $p \in X$ and a number $\varepsilon>0$, the $\varepsilon$-neighborhood of $p$ is $N(p, \varepsilon)=\{q \mid \rho(p, q)<\varepsilon\}$. If $A \subset X$ the diameter of $A$ is denoted by $\operatorname{diam} A$; $\operatorname{diam} A=\sup _{x, y \in A} \rho(x, y)$. If $Q$ is a compact space and $f_{1}$ and $f_{2}$ are maps of $Q$ into a metric space $X$, we define the distance between $f_{1}$ and $f_{2}$ by $\rho\left(f_{1}, f_{2}\right)=\sup _{x \in Q} \rho\left(f_{1}(x), f_{2}(x)\right)$.

If $M$ is an $n$-manifold the terms Bd $M$ and Int $M$ represent the sets of boundary and interior points respectively. The unit $n$-cell in Euclidean $n$-space is denoted $B^{n}$; the unit $(n-1)$-sphere $\mathrm{Bd} B^{n}=S^{n-1}$. Let $X$ be a metric space, $A \subset X, p \in$ $\operatorname{cl}(X-A)$. Then $X-A$ is $k$-LC at $p$ if for every $\varepsilon>0$ there is some $\delta>0$ such that each map $f: S^{k} \rightarrow N(p, \delta)-A$ extends to a map $g: B^{k+1} \rightarrow N(p, \varepsilon)-A$. Often $X$ is said to be locally simply connected if $X$ is 1-LC at each point $p \in X$.

Finally, suppose that $X$ and $Y$ are metric spaces endowed with metrics $\rho_{1}$ and $\rho_{2}$ respectively. Then a product metric $\rho$ on $X \times Y$ is induced by $\rho_{1}$ and $\rho_{2}$ according to the rule $\rho\left(\left(x_{1}, y_{1}\right),\left(x_{2}, y_{2}\right)\right)=\max \left\{\rho_{1}\left(x_{1}, x_{2}\right), \rho_{2}\left(y_{1}, y_{2}\right)\right\}$. We will refer to the canonical projection maps from $X \times Y$ onto $X$ and $Y$ respectively as $\pi_{1}$ and $\pi_{2}$.

Received by the editors August 6, 1979 and, in revised form, April 22, 1980.

AMS (MOS) subject classifications (1970). Primary 57A15; Secondary 54B10, 54B15, 54 F65.

Key words and phrases. Cell-like map, disjoint disks property, $n$-manifold, 1-LC, triangulation, locally simply connected. 
3. Mapping a disk into product spaces. There are two essential steps in our techinque. First we obtain mappings of the unit 2-cell $B^{2}$ into a product $X \times Y$ having relatively simple intersections with certain subspaces of the form $J_{i} \times Y, J_{i}$ a simple closed curve in $X$. Then we adjust mappings of $B^{2}$ into $J_{i} \times Y$ using the following lemma.

LEMMA 1. Let $Y$ be a locally compact, locally simply connected metric space such that no 1-dimensional subset of $Y$ contains an open subset of $Y$ and, for each arc $A$ in $Y, Y-A$ is $0-L C$ at each point of $A$. Let $A_{1}, \ldots, A_{k}$ be arcs in $S^{1}, x_{1}, \ldots, x_{k}$ distinct points in $Y, \varepsilon$ a positive number, $K$ a finite 2-complex, and $f$ a map of $K$ into $S^{1} \times Y$. Then there exists a map $f^{\prime}: K \rightarrow S^{1} \times Y$ such that

(1) $\rho\left(\pi_{2} f, \pi_{2} f^{\prime}\right)<\varepsilon$, and

(2) $f^{\prime}(K) \cap\left(\cup_{i=1}^{k}\left(A_{i} \times\left\{x_{i}\right\}\right)\right)=\varnothing$.

Moreover, if $C$ is a closed subset of $K-f^{-1} \pi_{2}^{-1}\left(\left\{x_{0}, \ldots, x_{k}\right\}\right)$, then $f^{\prime}$ can be obtained satisfying

(3) $f^{\prime}|C=f| C$.

Proof. We content ourselves with a sketch since the details are relatively routine. First we triangulate $K$ by a triangulation $T$ with fine mesh so that the 1-skeleton $T^{(1)}$ may be regarded as the union of simple closed curves which map under $f$ into small open sets in $S^{1} \times Y$. In fact, we may suppose that the image under $f$ of each 2-simplex of $T$ intersects at most one of the arcs $A_{i} \times\left\{x_{i}\right\}$ and any such image intersecting $f(C)$ misses all of the arcs $A_{i} \times\left\{x_{i}\right\}$. Next, we approximate $f$ by a map $f_{1}$ such that $\pi_{2} f_{1}\left(T^{(1)}\right) \subset Y-\left\{x_{1}, \ldots, x_{k}\right\}$. Then, altering only the $S^{1}$-coordinates of points of $f_{1}(K)$, we replace the images of 2-simplexes near the $\operatorname{arcs} A_{i} \times\left\{x_{i}\right\}$ in the following manner.

For each $\sigma_{j} \in T^{(2)}$ such that $f_{1}\left(\sigma_{j}\right) \cap\left(A_{i} \times\left\{x_{i}\right\}\right) \neq \varnothing$ for some $i$, let $N$ be an annular neighborhood of $\mathrm{Bd} \sigma_{j}$ in $\sigma_{j}$ such that $\pi_{2} f_{1}(N) \subset Y-\left\{x_{1}, \ldots, x_{k}\right\}$. Let $J$ denote the boundary component of $N$ in the interior of $\sigma_{j}$. Using a contraction of $\pi_{1} f_{1}\left(\mathrm{Bd} \sigma_{j}\right)$ in $S^{1}$ we obtain a map $g: N \rightarrow S^{1}$ such that $g(J)$ is a point in $S^{1}-A_{i}$ and $g\left|\operatorname{Bd} \sigma_{j}=\pi_{1} f_{1}\right| \operatorname{Bd} \sigma_{j}$. Then we define $f^{\prime}$ on $\sigma_{j}$ by the rule

$$
f^{\prime}(x)= \begin{cases}\left(g(x), \pi_{2} f_{1}(x)\right) & \text { if } x \in N, \\ \left(g(J), \pi_{2} f_{1}(x)\right) & \text { if } x \in \sigma_{j}-N .\end{cases}
$$

Property (1) depends only on restrictions imposed on $f_{1}$ since $\pi_{2} f^{\prime}=\pi_{2} f_{1}$. Property (2) is a consequence of three facts: that $\pi_{2} f_{1}\left(\sigma_{j}\right)$ contains at most one point of $\left\{x_{1}, \ldots, x_{k}\right\}$ for each $\sigma_{j}$; that $\pi_{2} f_{1}(N) \subset Y-\left\{x_{1}, \ldots, x_{k}\right\}$; and that $g(J) \subset S^{1}$ - $A_{i}$. Property (3) holds because $f^{\prime}=f$ on all simplexes of $K$ which intersect $C$.

THEOREM 2. Let $X$ and $Y$ be locally compact, locally simply connected metric spaces such that no 1-dimensional subset of either space contains an open subset and, for each arc in $X$ (or $Y), X-A(Y-A)$ is $0-L C$ at each point of $A$. Then $X \times Y$ has the disjoint disks property.

Proof. Let $\rho_{1}, \rho_{2}$ be metrics on $X$ and $Y$ respectively; $\rho$ the product metric on $X \times Y$ induced by $\rho_{1}$ and $\rho_{2}$. Let $f_{1}$ and $f_{2}$ be maps from $B^{2}$ into $X \times Y, \varepsilon$ a 
positive number. A first objective is to obtain approximations $f_{1}^{\prime}$ and $f_{2}^{\prime}$ of $f_{1}$ and $f_{2}$ respectively such that $f_{1}^{\prime}\left(B^{2}\right) \cap f_{2}^{\prime}\left(B^{2}\right)$ lies in the union of finitely many 1-dimensional continua in distinct levels $X \times\left\{y_{i}\right\}$.

By the local homotopy assumptions on $X$ and $Y$, there exist for each $i=1,2$ maps $\varphi_{i}: B^{2} \rightarrow X$ and $\theta_{i}: B^{2} \rightarrow Y$ and a triangulation $T_{0}$ of $B^{2}$ such that

(1) diam $f_{i}\left(\sigma_{j}\right)<\varepsilon / 9$ for each simplex $\sigma_{j}$ of $T_{0}$,

(2) $\rho\left(\varphi_{i}, \pi_{1} f_{i}\right)<\varepsilon / 9$,

(3) $\rho\left(\theta_{i}, \pi_{2} f_{i}\right)<\varepsilon / 3$,

(4) $\varphi_{i} \mid T_{0}^{(1)}$ and $\theta_{i} \mid T_{0}^{(1)}$ are embeddings, and

(5) $\varphi_{1}\left(T_{0}^{(1)}\right) \cap \varphi_{2}\left(T_{0}^{(1)}\right)=\varnothing$.

(Here we are using $T_{0}^{(1)}$ to denote the space underlying $T_{0}^{(1)}$ as well as the simplicial complex consisting of all 1-simplexes and vetices of $T_{0}$.)

For each 2-simplex $\sigma_{j} \in T_{0}$ choose $y_{1, j} \in \theta_{1}\left(\sigma_{j}\right), y_{2, j} \in \theta_{2}\left(\sigma_{j}\right)$. These points must be chosen so that $y_{i, j} \neq y_{p, q}$ if either $i \neq p$ or $j \neq q$. Let $F$ denote the set of such points $y_{i, j}$. For each $i=1,2$ there exist homotopies $H_{i, j}: \operatorname{Bd} \sigma_{j} \times[0,1] \rightarrow \theta_{i}\left(\sigma_{j}\right)$ such that $H_{i, j}(p, 0)=\theta_{i}(p)$ and $H_{i, j}(p, 1)=y_{i, j}$ for each $p \in \operatorname{Bd} \sigma_{j}$. Now, choose a neighborhood $N_{j}$ of $\mathrm{Bd} \sigma_{j}$ in $\sigma_{j}$ homeomorphic to $\mathrm{Bd} \sigma_{j} \times[0,1]$. Let $\alpha_{j}$ be a homeomorphism from $N_{j}$ onto $\operatorname{Bd} \sigma_{j} \times[0,1]$ which sends each $s$ in $\operatorname{Bd} \sigma_{j}$ to $(s, 0)$ in $\mathrm{Bd} \sigma_{j} \times[0,1]$; let $\beta_{j}$ be a map from $\sigma_{j}$ onto $\sigma_{j}$ such that $\beta_{j} \mid N_{j}$ is a retraction onto $\operatorname{Bd} \sigma_{j}$ and $\beta_{j} \mid \operatorname{cl}\left(\sigma_{j}-N_{j}\right)$ is a homeomorphism. For each $i=1,2$ and approximation $f_{i}^{\prime}$ is defined simplex by simplex by the rule

$$
\left(f_{i}^{\prime} \mid \sigma_{j}\right)(p)= \begin{cases}\left(\varphi_{i} \beta_{j}(p), H_{i, j} \alpha_{j}(p)\right) & \text { if } p \in N_{j}, \\ \left(\varphi_{i} \beta_{j}(p), y_{i, j}\right) & \text { if } p \in \sigma_{j}-N_{j} .\end{cases}
$$

Each map $f_{1}^{\prime}$ defined in this way satisfies the following:

(6) for each 2-simplex $\sigma_{j}$ in $T_{0}, f_{1}^{\prime}\left(\sigma_{j}\right) \cap f_{2}^{\prime}\left(B^{2}\right) \subset\left(\varphi_{2}\left(T_{0}^{(1)}\right) \times\left\{y_{1, j}\right\}\right) \cup$ $\left[\cup_{k}\left(\varphi_{1}\left(\mathrm{Bd} \sigma_{j}\right) \times\left\{y_{2, k}\right\}\right)\right]$

(7) for each 2-simplex $\sigma_{j}$ in $T_{0}, f_{2}^{\prime}\left(\sigma_{j}\right) \cap f_{1}^{\prime}\left(B^{2}\right) \subset\left(\varphi_{1}\left(T_{0}^{(1)}\right) \times\left\{y_{2, j}\right\}\right) \cup$ $\left[\cup_{k}\left(\varphi_{2}\left(\mathrm{Bd} \sigma_{j}\right) \times\left\{y_{1, k}\right\}\right)\right]$

(8) $\pi_{1} f_{i}^{\prime}\left(\sigma_{j}\right)=\varphi_{i}\left(\sigma_{j}\right)$ for each $i$;

(9) $\pi_{2} f_{i}^{\prime}\left(\sigma_{j}\right)=\theta_{i}\left(\sigma_{j}\right)$ for each $i$

(10) $\pi_{1} f_{i}^{\prime}\left|T_{0}^{(1)}=\varphi_{i}\right| T_{0}^{(1)}$ for each $i$; and

(11) $\pi_{2} f_{i}^{\prime}\left|T_{0}^{(1)}=\theta_{i}\right| T_{0}^{(1)}$ for each $i$.

Our next step is to modify each $f_{i}^{\prime}$ slightly in the factor $Y$, obtaining maps $f_{1}^{\prime \prime}$ and $f_{2}^{\prime \prime}$ from $B^{2}$ into $X \times Y$ such that, for each 2-simplex $\sigma_{j}$ in $T_{0}$,

$$
\left[f_{1}^{\prime \prime}\left(B^{2}\right) \cap\left(\varphi_{2}\left(\mathrm{Bd} \sigma_{j}\right) \times Y\right)\right] \cup\left[f_{2}^{\prime \prime}\left(B^{2}\right) \cap\left(\varphi_{1}\left(\mathrm{Bd} \sigma_{j}\right) \times Y\right)\right]
$$

lies within the finite union of arcs in distinct levels $\left[\varphi_{1}\left(\operatorname{Bd} \sigma_{j}\right) \cup \varphi_{2}\left(\operatorname{Bd} \sigma_{j}\right)\right] \times\left\{z_{k}\right\}$. To this end choose triangulations $T_{1}, T_{2}, T_{3}, \ldots$ such that $T_{i}$ subdivides $T_{i-1}$ for each $i$ and $\operatorname{limit}_{i \rightarrow \infty}$ (mesh $\left.T_{i}\right\}=0$. Again, by the use of the local homotopy assumption on $X$, approximate each $\varphi_{i}$ by a map $\psi_{i}, i=1,2$, such that

(12) $\rho_{1}\left(\psi_{i}, \varphi_{i}\right)<\varepsilon / 3$,

(13) $\psi_{1}\left(T_{j}^{(1)}\right) \cap \varphi_{2}\left(T_{0}^{(1)}\right)=\varnothing$ for each $j=1,2,3, \ldots$,

(14) $\psi_{2}\left(T_{j}^{(1)}\right) \cap \varphi_{1}\left(T_{0}^{(1)}\right)=\varnothing$ for each $j$, and

(15) $\psi_{i}\left|T_{0}^{(1)}=\varphi_{i}\right| T_{0}^{(1)}$. 
Choose $\delta>0$ such that no subset of $X \times Y$ having diameter less than $\delta$ contains a simple closed curve of the form $\varphi_{i}\left(\mathrm{Bd} \sigma_{j}\right) \times\{y\}, \sigma_{j} \in T_{0}^{(2)}, y \in Y$. Let $m$ be a positive integer such that

(16) diam $\psi_{i}\left(\tau_{k}\right)<\delta$ for each simplex $\tau_{k}$ of $T_{m}, i=1,2$.

For each 2-simplex $\sigma_{j} \in T_{0}$ let $U_{1, j}$ and $U_{2, j}$ be path-connected open neighborhoods of

$$
y_{1, j}=\pi_{2}\left(f_{1}^{\prime}\left(\sigma_{j}\right)-\left(\varphi_{1}\left(\operatorname{Bd} \sigma_{j}\right) \times Y\right)\right) \text { and } y_{2, j}=\pi_{2}\left(f_{2}^{\prime}\left(\sigma_{j}\right)-\left(\varphi_{2}\left(\operatorname{Bd} \sigma_{j}\right) \times Y\right)\right)
$$

respectively such that

(17) $\operatorname{diam} U_{i, j}<\varepsilon / 3$.

Assume in addition that $U_{i, j} \cap U_{p, q}=\varnothing$ unless $i=p, j=q$. Let $\tau_{j, 1}, \ldots, \tau_{j, r(j)}$ denote the 2-simplexes of $T_{m}$ lying in $\sigma_{j}$. Let $z_{1, j, 1}, \ldots, z_{1, j, s(j)}$ be distinct points of $U_{1, j}$; let $z_{2, j, 1}, \ldots, z_{2, j, s(j)}$ be distinct points of $U_{2, j}$ missing $\theta_{1}\left(T_{0}^{(1)}\right) \cup \theta_{2}\left(T_{0}^{(1)}\right) \cup F$. As the choice of $\sigma_{j}$ varies, the total collection $F^{\prime}$ of points $z_{i, j, k}$ engendered by the subdivision $T_{m}$ must have the property $z_{i, j, k} \neq z_{p, q, r}$ whenever $i \neq p, j \neq q$, or $k \neq r$. Since $U_{1, j}$ and $U_{2, j}$ are path-connected there exists for each $i, j$, and $k=$ $1, \ldots, s(j)$ a homotopy $H_{i, j, k}: \operatorname{Bd} \tau_{j, k} \times[0,1] \rightarrow U_{i, j}$ such that $H_{i, j, k}\left(\mathrm{Bd} \tau_{k} \times\{0\}\right)$ $=y_{i, j}$ and $H_{i, j, k}\left(\mathrm{Bd} \tau_{k} \times\{1\}\right)=z_{i, j, k}$. By making use of the technique used to define $f_{1}^{\prime}$ and $f_{2}^{\prime}$ we obtain maps $\mu_{1, j}$ and $\mu_{2, j}$ from each 2-simplex $\sigma_{j}$ of $T_{0}$ into $X \times Y$ such that

(18) for each $\tau_{j, k} \in T_{m}^{(2)} \cap \sigma_{j}, \mu_{i, j}\left(\tau_{j, k}\right) \subset\left[\psi_{i}\left(\operatorname{Bd} \tau_{j, k}\right) \times U_{i, j}\right] \cup\left[\psi_{i}\left(\tau_{j, k}\right) \times\left\{z_{i, j, k}\right\}\right]$, and

(19) $\pi_{1} \mu_{i, j}\left|\left(T_{m}^{(1)} \cap \sigma_{j}\right)=\psi_{i}\right|\left(T_{m}^{(1)} \cap \sigma_{j}\right)$.

The promised approximations $f_{i}^{\prime \prime}$ are defined simplex by simplex by the rule

$$
f_{i}^{\prime \prime}(p)= \begin{cases}f_{i}^{\prime}(p) & \text { if } p \in N_{j}, \\ \mu_{i, j} \beta_{j}(p) & \text { if } p \in \sigma_{j}-N_{j} .\end{cases}
$$

Since $z_{1, j, k} \neq z_{2, p, q}$ for all values $j, k, p$, and $q$, property (18) implies that $\left[f_{1}^{\prime \prime}\left(B^{2}\right)-\right.$ $\left.\left(\varphi_{1}\left(T_{0}^{(1)}\right) \times Y\right)\right] \cap\left[f_{2}^{\prime \prime}\left(B^{2}\right)-\left(\varphi_{2}\left(T_{0}^{(1)}\right) \times Y\right)\right]=\varnothing$. Thus, by (13), (16), and (18), for each $\sigma_{j} \in T_{0}^{(2)}, f_{1}^{\prime \prime}\left(B^{2}\right) \cap\left(\varphi_{2}\left(B d \sigma_{j}\right) \times Y\right)$ is confined to the union of finitely many $\operatorname{arcs} A_{1, j, k} \times\left\{z_{1, j, k}\right\}, A_{1, j, k} \subset \varphi_{2}\left(\mathrm{Bd} \sigma_{j}\right)$. Similarly, by (14), (16), and (18), $f_{2}^{\prime \prime}\left(B^{2}\right) \cap$ $\left(\varphi_{1}\left(\mathrm{Bd} \sigma_{j}\right) \times Y\right)$ is confined to the union of finitely many $\operatorname{arcs} A_{2, j, k} \times\left\{z_{2, j, k}\right\}$, $A_{2, j, k} \subset \varphi_{1}\left(\mathrm{Bd} \sigma_{j}\right)$.

We complete the proof by applying Lemma 1 to approximate each map $f_{i}^{\prime \prime} \mid N_{j}$ by a map $\chi_{i, j}: N_{j} \rightarrow \varphi_{i}\left(\mathrm{Bd} \sigma_{j}\right) \times Y$ such that

(20) $\rho\left(\pi_{2} \chi_{i, j}, \pi_{2} f_{i}^{\prime \prime} \mid N_{j}\right)<\varepsilon / 3$,

(21) $\chi_{1, j}\left(N_{j}\right) \cap f_{2}^{\prime \prime}\left(B^{2}\right)=\varnothing$,

(22) $\chi_{2, j}\left(N_{j}\right) \cap f_{1}^{\prime \prime}\left(B^{2}\right)=\varnothing$, and

(23) $\chi_{i, j}\left|\operatorname{Bd} N_{j}=f_{i}^{\prime \prime}\right| \operatorname{Bd} N_{j}=f_{i}^{\prime} \mid \operatorname{Bd} N_{j}$.

Using these maps we define two maps $f_{i}^{\prime \prime \prime}: B^{2} \rightarrow X \times Y(i=1,2)$ by putting $f_{i}^{\prime \prime \prime}=\chi_{i, j}$ on each annulus $N_{j}$ and by putting $f_{i}^{\prime \prime \prime}=f^{\prime \prime}$ on $B^{2}-\left(\cup_{j} N_{j}\right)$. Properties (21), (22), and (23) clearly imply that $f_{1}^{\prime \prime \prime}\left(B^{2}\right) \cap f_{2}^{\prime \prime \prime}\left(B^{2}\right)=\varnothing$. It remains only to check that $\rho\left(f_{i}, f_{i}^{\prime \prime \prime}\right)<\varepsilon$.

Suppose that $p \in N_{j}$ for some $j$. Then, conditions (1) and (2) imply that 
diam $\varphi_{i}\left(\mathrm{Bd} \sigma_{j}\right)<\varepsilon / 3$. This fact combined with (20) implies that $\rho\left(f_{i}^{\prime \prime \prime}(p), f_{i}^{\prime \prime}(p)\right)<$ $\varepsilon / 3$. Thus $\rho\left(f_{i}^{\prime \prime \prime}(p), f_{i}(p)\right)<\varepsilon / 3+\rho\left(f_{i}^{\prime \prime}(p), f_{i}(p)\right)$. Moreover, $f_{i}^{\prime \prime}(p)=f_{i}^{\prime}(p)$ and $\pi_{1} f_{i}^{\prime}(p)=\varphi_{1}(q)$ for some $q \in \sigma_{j}$ by (8). Thus condition (1) and (2) yield

$$
\rho\left(\pi_{1} f_{i}^{\prime \prime}(p), \pi_{1} f_{i}(p)\right)<\varepsilon / 3+\varepsilon / 3 .
$$

By (9) we have $\pi_{2} f_{i}^{\prime}(p)=\theta_{i}\left(q^{\prime}\right)$ for some $q^{\prime} \in \sigma_{j}$. Conditions (1) and (3) yield

$$
\rho\left(\pi_{2} f_{i}^{\prime \prime}(p), \pi_{2} f_{i}(p)\right)<\varepsilon / 3+\varepsilon / 3 .
$$

Combining these we have $\rho\left(f_{i}^{\prime \prime \prime}(p), f_{i}(p)\right)<\varepsilon$.

Now, suppose that $p \in \sigma_{j}-N_{j}$ for some $j$. Thus, $f_{i}^{\prime \prime \prime}(p)=f_{i}^{\prime \prime}(p)$. Property (18) implies that $\pi_{1} f_{i}^{\prime \prime}(p)=\psi_{i}(q)$ for some $q \in \sigma_{j}$. Conditions (1), (2), and (12) yield

$$
\rho\left(\psi_{i}(q), \pi_{1} f_{i}(p)\right)<\varepsilon .
$$

Conditions (17) and (18) yield

$$
\rho\left(\pi_{2} f_{i}^{\prime \prime}(p), \pi_{2} f_{i}(p)\right)<\varepsilon / 3+\rho\left(\pi_{2} f_{i}^{\prime}(p), \pi_{2} f_{i}(p)\right) .
$$

By (9), $\pi_{2} f_{i}^{\prime}(p)=\theta_{i}\left(q^{\prime}\right)$ for some $q^{\prime} \in \sigma_{j}$. Thus (1) and (3) yield

$$
\rho\left(\pi_{2} f_{i}^{\prime}(p), \pi_{2} f_{i}(p)\right)<\varepsilon / 3+\varepsilon / 3 .
$$

Combining the above gives $\rho\left(f_{i}^{\prime \prime \prime}(p), f_{i}(p)\right)<\varepsilon$ as required. This completes the proof of Theorem 2 .

4. Products of cell-like images of manifolds. The following result gives abundant examples of factorizations of certain manifolds into nonmanifold factors.

COROLlary 3. Let $M, N$ be finite-dimensional manifolds of dimension at least three without boundary. Let $P_{1}$ and $P_{2}$ be proper cell-like maps from $M$ and $N$ respectively onto topological spaces $X$ and $Y$. If $X$ and $Y$ are ANR's then $X \times Y$ is homeomorphic to $M \times N$.

Proof. Since $P_{1}$ and $P_{2}$ are proper cell-like maps the product $P_{1} \times P_{2}: M \times N$ $\rightarrow X \times Y$ is a proper cell-like map as well. Since $X$ and $Y$ are ANR's, $X \times Y$ is an ANR. Furthermore, $X$ and $Y$ are generalized manifolds by [9]. It follows from Lemma 2.1 and Proposition 2.2 of [6] that $X$ and $Y$ satisfy the hypotheses of Theorem 2. Thus, Edwards' approximation theorem [7] implies that $P_{1} \times P_{2}$ is approximable by homeomorphisms.

It is presently unknown whether the prior result remains true in case $\operatorname{dim} N=1$. If $\operatorname{dim} M=\operatorname{dim} N=2$, the result is classical. In case $\operatorname{dim} M>3, \operatorname{dim} N=2$, the result is due to $\mathbf{R}$. J. Daverman.

\section{REFERENCES}

1. W. P. Amsbury, The product of null-sequence arc-decompositions is Euclidean, Ph.D. thesis, Univ. of Tennessee, 1972.

2. R. H. Bing, The cartesian product of a certain nonmanifold and a line is $E^{4}$, Ann. of Math. (2) 70 (1959), 399-412.

3. A. J. Boals, Non-manifold factors of euclidean spaces, Fund. Math. 68 (1970), 159-177. 
4. J. W. Cannon, Shrinking cell-like decompositions of manifolds. Codimension three, Ann. of Math. (2) 110 (1979), 83-112.

5. 832-864.

6. R. J. Daverman, Detecting the disjoint disks property, Pacific J. Math. (to appear).

7. R. D. Edwards, The topology of manifolds and cell-like maps, Proc. Internat. Congress Math., 1978 Academia Scientarium Fennica, Helsinki, 1980, pp. 111-127.

8. K. W. Kwun, Product of euclidean spaces modulo an arc, Ann. of Math. (2) 79 (1964), $104-107$.

9. R. L. Wilder, Monotone mappings of manifolds, Pacific J. Math. 7 (1957), 1519-1528.

Department of Mathematics, Pembroke State University, Pembroke, North Carolina 28372 